\title{
Thin-layer chromatography on polyacrylonitrile
}

\section{Investigation of the separation mechanisms for tris- (alkylxanthato)cobalt(III) complexes}

\author{
T. J. Janjić*, D. M. Milojković, G. N. Vučković and M. B. Ćelap \\ Faculty of Chemistry, University of Belgrade, Studentski trg 16, P.O. Box 550, 11001 Belgrade (Yugoslavia)
}

(First received September 24th, 1991; revised manuscript received December 16th, 1991)

\begin{abstract}
The chromatographic behaviour of six tris(alkylxanthato)cobalt(III) complexes on polyacrylonitrile sorbent thin layers was investigated using four non-aqueous and two aqueous solvent systems. It was assumed that their separation under normal-phase conditions was achieved by the mechanism of hydrogen-bond formation between highly electronegative sorbate oxygen and/or sulphur atoms and sorbent methync group hydrogens. In contrast, it was assumed that under reversed-phasc conditions the separation was based on non-specific hydrophobic interactions. Finally, the linear dependence between the number of carbon atoms in the ligands and the corresponding $\mathbf{R}_{M}$ values of the investigated complexes of a homologous series was confirmed.
\end{abstract}

\section{INTRODUCTION}

A polyacrylonitrile sorbent (PANS), the preparation and characteristics of which were described in the first paper of this series [1], was subsequently applied to the separation of cis-trans isomeric cobalt(III) complexes [1], foodstuff dyes [2] and tris $(\beta$ diketonato) complexes of cobalt(III), chromium (III) and ruthenium(III) [3]. The separation mechanisms for these compounds were considered, and it was also shown that PANS, owing to its moderate polarity [2], can be applied to the separation of different classes of compounds by both normal- and reversed-phase chromatography.

Continuing these investigations, in this work we investigated the chromatographic behaviour of six tris(alkylxanthato)cobalt(III) complexes on thin layers of PANS with non-aqueous and aqueous solvent systems. Among other things, we wanted to check whether the earlier established rule of a linear dependence between the number of carbon atoms in the ligands and the corresponding $R_{M}$ values of a homologous series of metal complexes, obtained on thin layers of silica gel [4-6], as well as in the paper chromatography $[7,8]$, is valid for the separations on PANS. In addition, we considered the separation mechanisms for the investigated complexes.

\section{EXPERIMENTAL}

The complexes were synthesized by the procedure described previously [6]. The preparation of PANS, its application to microscope slides and the development of chromatograms by the ascending method were performed as described in Part I [1].

The chromatographic plates were spotted with $0.2-\mu 1$ aliquots of freshly prepared solutions $(2 \mathrm{mg}$ $\mathrm{cm}^{-3}$ ) of the complexes in acetone. Before development, the spotted plates were equilibrated for 30 min in a chromatographic chamber saturated with vapour of the solvent system being used. All solvents used were of analytical-reagent grade; the compositions of the solvent systems and development times are listed in Table I. After development, 
TABLE I

COMPOSITION OF THE CHROMATGGRAPHIC SOLVENT SYSTEMS USED AND THE CORRESPONDING DEVELOPMENT TIMES

\begin{tabular}{lll}
\hline No. & Solvent system (v/v) & $\begin{array}{l}\text { Development } \\
\text { time (min) }\end{array}$ \\
\hline 1 & $n$-Hexane & 25 \\
2 & $n$-Nonane & 25 \\
3 & $n$-Hexane-carbon tetrachloride $(90: 10)$ & 25 \\
4 & $n$-Hexane-benzene $(90: 10)$ & 25 \\
5 & Acetone-water $(80: 20)$ & 60 \\
6 & Tetrahydrofuran-water $(70: 30)$ & 100 \\
\hline
\end{tabular}

the coloured spots of the complexes were readily visible.

\section{RESULTS AND DISCUSSION}

The results of chromatographic separations are given in Table II. As can be seen from the application of two one-component and two poly-component non-aqueous solvent systems, the retention of the chelates decreased with increasing size of the $n$-alkyl chain of the ligands. However, with aqueous solvent systems the sequence of $R_{\boldsymbol{H}}$. values was reversed. In all instances a linear dependence of the corresponding $R_{M}$ values on the number of carbon atoms in the $n$-alkyl chain of the ligands was observed (Fig. 1). As the absolute values of the slopes of these straight lines correspond to the logarithm of the separation factor [10] of two neighbouring

\section{TABLE II}

$h R_{F}$ VALUES IN SOLVENTS $1-6^{a}$ OF THE INVESTIGATED TRIS(ALKYLXANTHATO)COBALT(III) COMPLEXES AND THE CORRESPONDING TAFT'S INDUCTIVE CONSTANTS $\left(\sigma^{*}\right)$ FOR ALKYL SUBSTITUENTS [9]

\begin{tabular}{|c|c|c|c|c|c|c|c|c|}
\hline \multirow[t]{2}{*}{ No. } & \multirow{2}{*}{$\begin{array}{l}\text { Alkyl } \\
\text { group }\end{array}$} & \multicolumn{6}{|c|}{$h R_{F}$} & \multirow[t]{2}{*}{$\sigma^{*}$} \\
\hline & & 1 & 2 & 3 & 4 & $\leqslant$ & 6 & \\
\hline 1 & Methyl & 21 & 18 & 27 & 47 & 71 & 60 & 0.000 \\
\hline 2 & Ethyl & 36 & 27 & 42 & 67 & 62 & 51 & -0.100 \\
\hline 3 & n-Propyl & 48 & 41 & 63 & 84 & 48 & 42 & -0.115 \\
\hline 4 & n-Butyl & 60 & 57 & 73 & 93 & 39 & 34 & -0.130 \\
\hline 5 & Isobutyl & 62 & 60 & 78 & 95 & 37 & 32 & -0.125 \\
\hline 6 & sec.-Butyl & 59 & 55 & 69 & 93 & 43 & 43 & -0.210 \\
\hline
\end{tabular}

${ }^{a}$ See Table I.

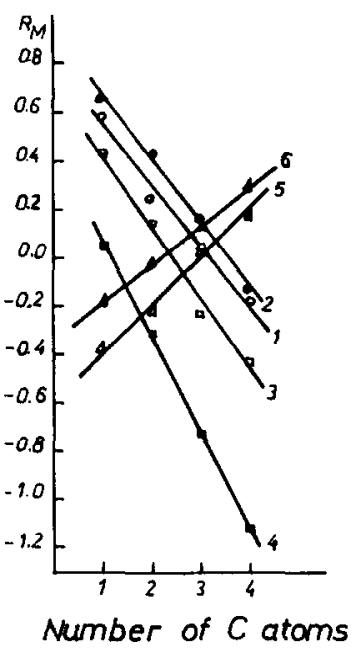

Fig. 1. Dependence of $R_{M}$ values of the investigated tris( $n$-alkylxanthato)cobalt(III) complexes on the number of carbon atoms in the ligand. The numbers on the lines refer to the solvent sys tem in Table $\mathbf{I}$.

members of a series, it may be concluded that this factor was higher under normal-phase conditions when two-component solvent systems were used in comparison with the values obtained with one-component ones. Under reversed-phase conditions, this factor was the smallest. Hence it was established that the highest separation factor was obtained with solvent $4(\alpha=2.5)$. In this connection, it is interesting that the highest $\alpha$ value for the same homologous series obtained on silica gel [6] was 1.6.

The $R_{F}$ values of the isomeric tris(butylxanthato)cobalt(III) complexes, obtained under the conditions of normal-phase chromatography (by application of non-aqueous solvent systems) (Table II, solvents 1-4) were found to increase in the order of the corresponding Co(III) complexes: sec-butylxanthate $<n$-butylxanthate $<$ isobutylxanthate, although the differences in $R_{F}$ values are relatively small. When the chromatographic separations of these complexes were performed by application of aqueous solvent systems (reversed-phase chromatography), a reversed sequence for these complexes was obtained (Table II).

As reported previously [11], under conditions of normal-phase chromatography, the saturated chelates are most often adsorbed by the mechanism of hydrogen-bond formation between the solute and a 
sorbent. The sorption of the investigated tris(alkylxanthato)cobalt(III) complexes under the conditions of normal-phase chromatography on thin layers of PANS may also be explained by the mechanism of hydrogen-bond formation, i.e., between oxygen and/or sulphur chelate atoms [12] and methyne hydrogen atoms of the sorbent [13]. As is known, the strength of hydrogen bonds formed depends on the electron density on the corresponding electronegative atoms; in our case a positive inductive effect of alkyl groups in alkylxanthato ligands would cause a decrease in the $R_{F}$ values of complexes with increasing size of the $n$-alkyl side-chain. However, the oppositie results were obtained (Table II, solvent systems 1-4), which means that in the process of chromatographic separation the mobility of the chelate depends mainly on other factors. This
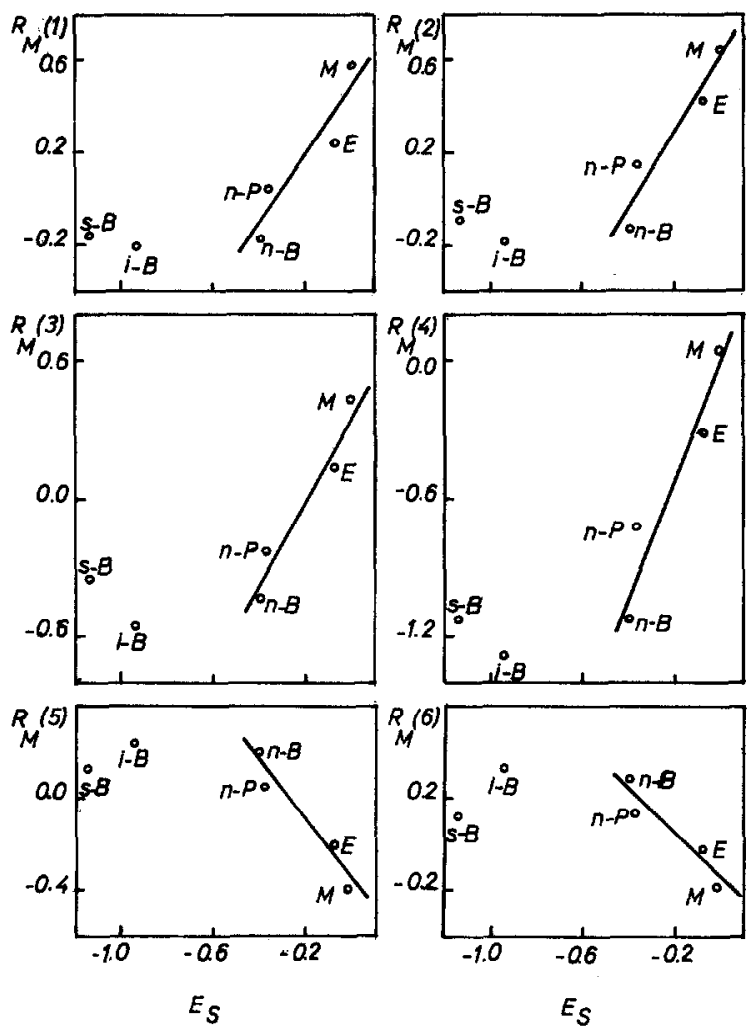

Fig. 2. Dependence of $R_{M}$ values of the investigated tris(alkylxanthato)cobalt(III) complexes on the corresponding side-chain steric constants $\left(E_{\mathrm{s}}\right)$. The numbers in parentheses refer to the solvent systems in Table $\mathrm{I}$. $\mathbf{M}=$ Methyl; $\mathrm{E}=$ ethyl; $\mathrm{n}-\mathrm{P}=$ $n$-propyl; $\mathrm{n}-\mathrm{B}=n$-butyl; $\mathrm{i}-\mathrm{B}=$ isobutyl; $\mathrm{s}-\mathrm{B}=$ sec -butyl. might be steric hindrance, as stated by Snyder [14], analogous to the results obtained in chromatographic separations of some structurally similar metal dialkyldithiophosphates on silica get [15], or the solubilities of the investigated complexes in the corresponding solvents. In order to check which of the two mentioned effects is predominant, we plotted the $R_{M}$ values obtained versus the steric constants of the corresponding side-chains [15,16] (Fig. 2 ), and also versus the logarithm of the complex solubilities in $\mathrm{CCl}_{4}[6,17]$, which is the more polar component of one of the solvents used (Fig. 3). These data show in both instances a good correlation for $n$-alkylsubstituted complexes, i.e., both effects probably play important roles in their separation processes.

The results obtained for the investigated $n$-alkyl-
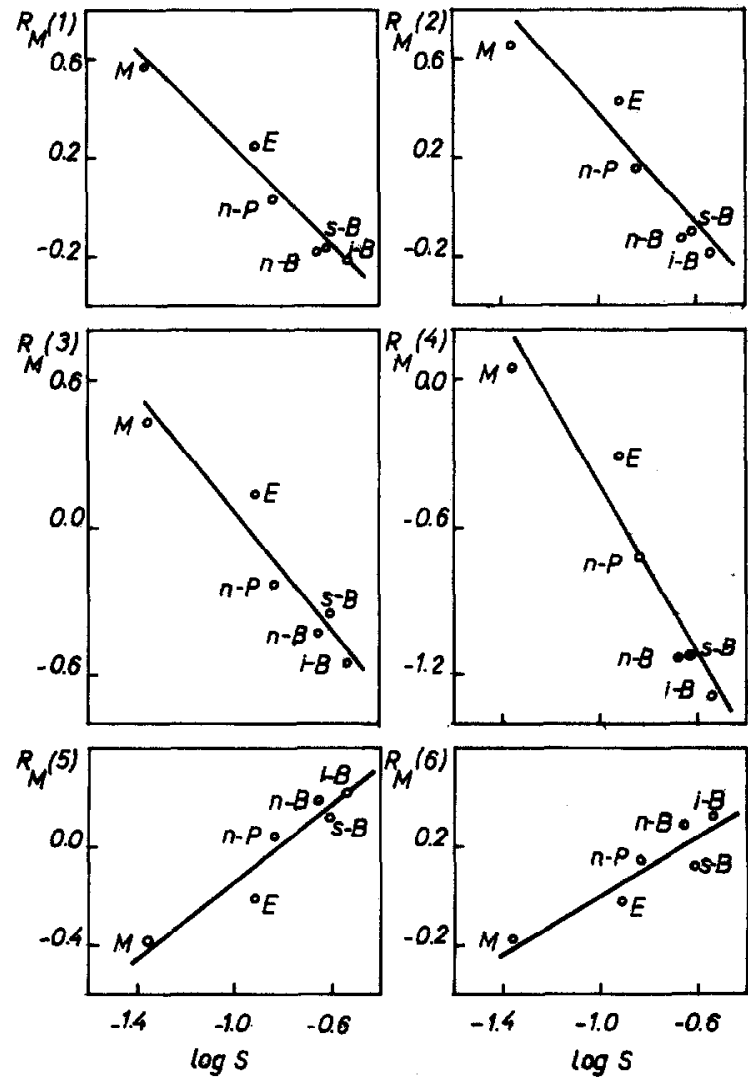

Fig. 3. Dependence of $R_{M}$ values of the investigated tris(alkylxanthato)cobalt(III) complexes on the complex solubility logarithm $(\log S)$ in $\mathrm{CCl}_{4}$. Numbers in parentheses and abbreviations as in Fig. 2. 
xanthato chelates under conditions of reversedphase chromatography (Table II, solvent systems 5 and 6) are in accordance with those expected, viz., with an increase in the $\boldsymbol{n}$-alkyl chain in the ligands, the hydrophobic moiety of a chelate acquires a greater surface area of contact with non-polar $-\mathrm{CH}_{2}-\mathrm{CH}$ segments of polyacrylonitrile, resulting in an increased retention of complexes. In addition, the order of the investigated alkylxanthato complexes under conditions of reversed-phase chromatography on PANS is analogous to that obtained by reversed-phase chromatography of a homologous series of these chelates on thin layers of some alkylmodified silica gels [18].

As already mentioned, the isomeris tris(butylxanthato)cobalt(III) complexes, regardless of the small differences obtained in $\boldsymbol{R}_{\boldsymbol{F}}$ values, exhibit the same sequence in normal-phase chromatography by application of all non-aqueous solvent systems used (Table II, solvent systems. 1-4). This sequence is analogous to the earlier established order of the same complexes on thin layers of silica gel [6], in accord with the hydrogen bond formation hypothesis [11]. Such behaviour of the isomeric butylxanthato complexes is not in accord with steric effects of the corresponding ligand side-chains. In addition, as seen from Fig. 2, regardless of large differences in steric effects of the isomeric butyl substituents, the differences in complex $R_{F}$ values obtained are very small. On the other hand, Fig. 3 shows that the order of $R_{M}$ values of the isomeric butylxanthato complexes is also not in accord with their solubilities. Therefore, we assume that in this instance the inductive effects of the corresponding ligand side-chains, which influence the electron density on ligand electro-negative atoms, play a decisive role, in accordance with the corresponding inductive constants of the isomeric butyl substitutens (Table II).

The reversed order of $R_{F}$ values for isomeric butylxanthato complexes obtained under reversedphase conditions (Table II, solvent systems 5 and 6 ), is in accordance with that expected, because in this instance specific interactions of the sorbate are acting towards polar molecules of the mobile phase.

On the basis of the above, it may be concluded that it has been established that lintear dependence between the number of carbon atoms in ligands and the corresponding $R_{M}$ values of a homologous series of metal complexes is also valid on PANS, in both normal- and reversed-phase chromatography. In addition, it is obvious that the dominant separation mechanism for the investigated chelates on PANS is hydrogen bond formation between the sorbates and the sorbent under normal-phase conditions and non-specific hydrophobic interactions in reversed-phase chromatography.

\section{ACKNOWLEDGEMENTS}

The authors are grateful to the Serbian Republic Research Fund for financial support and to $\mathrm{Mr}$. Žarko Arbutina for technical assistance.

\section{REFERENCES}

1 T. J. Janjić, D. M. Milojković, Ž. J. Arbutina, Ž. Lj. Tešić and M. B. Celap, J. Chromatogr., 481 (1989) 465.

2 T. J. Janjić, D. M. Milojković, Ž. J. Arbutina and M. B. Celap, J. Serb. Chem. Soc., 56 (1991) 33.

3 T. J. Janjić, D. M. Milojković, Ž. Lj. Tešić and M. B. Celap, J. Planar Chromatogr., 3 (1990) 495.

4 O. Liška, J. Lehotay, E. Branšteterova and G. Guiochon, $J$. Chromatogr., 171 (1979) 153.

5 M. B. Ćclap, G. Vučković, T. J. Janjić, M. J. Malinar and P. N. Radivojša, J. Chromatogr., 196 (1980) 59.

6 G. Vučković, N. Juranić and M. B. Ćlap, J. Chromatogr., 361 (1986) 217.

7 M. B. Ćelap, M. J. Malinar, S. Sarić, T. J. Janjić and P. N. Radivojša, J. Chromatogr., 153 (1978) 253.

8 T. J. Janjić, Ž. Lj. Tešić, M. J. Malinar, P. N. Radivojša and M. B. Celap, J. Chromatogr., 331 (1985) 273.

9 B. P. Nikolskii (Editor), Spravochnik Khimika, Vol. III, Khimiya, Moscow, 1964, p. 954.

10 K. Saitoh, M. Kobayashi and N. Suzuki, Anal. Chem., 53 (1981) 2309.

11 A. R. Timerbaev and O. M. Petrukhin, Zhidkostnaya Adsorbtsionnaya Khromatografiya Khelatov, Nauka, Moscow, 1989 , p. 26.

12 A. R. Timerbaev and O. M. Petrukkin, Zhidkostnaya Adsorbtsionnaya Khromatografija Khelatov, Nauka, Moscow, 1989 , p. 74.

13 Römpps Chemie-Lexikon, Franckh'sche Verlagshandlung, Stuttgart, 6th ed., 1966, Band III, p. 5021; 8th ed., 1987, Band V, p. 3274.

14 L. R. Snyder, in E. Heftmann (Editor), Chromatography, Reinhold, New York, 2nd ed., 1967, p. 70.

I5 A. R. Timerbaev, V. V. Salov and O. M. Petrukhin, 7h. Anal. Khim., 40 (1985) 237

16 B. P. Nikolskii (Editor), Spravochnik Khimika, Vol. III, Khimiya, Moscow, 1964, p. 956.

17 L. Saitoh, Bunseki Kagaku, 35 (1986) 895.

18 H. Eggers and H. A. Russel, Chromatographia, 17 (1983) 486 